PAPER

\title{
Theoretical analysis of the pathophysiology of syringomyelia associated with adhesive arachnoiditis
}

\author{
H S Chang, H Nakagawa
}

J Neurol Neurosurg Psychiatry 2004;75:754-757. doi: 10.1136/jnnp.2003.018671

See end of article for authors' affiliations

....................

Correspondence to:

Dr Han Soo Chang,

Department of

Neurological Surgery,

Aichi Medical University,

Aichi-gun 480-1195,

Japan; chang@

aichi-med-u.ac.jp

Received 16 May 2003

In revised form

15 August 2003

Accepted

22 September 2003

\begin{abstract}
Objective : To apply a theoretical model to analyse the derangement of cerebrospinal fluid (CSF) dynamics in syringomyelia associated with adhesive arachnoiditis.

Methods: An electrical circuit model of CSF dynamics in the spine was used. With this model, the derangement of CSF dynamics in adhesive arachnoiditis was simulated. The effects of various surgical procedures were then analysed, such as syringo-subarachnoid shunting, subarachnoid bypass, and foramen magnum decompression.

Results: When CSF flow in the subarachnoid space was obstructed at a certain point, the pressure inside the spinal cord increased in the segment immediately distal to the blockage. This location of increased pressure corresponded to the preferred site of syrinx formation in adhesive arachnoiditis. Syringosubarachnoid shunting, subarachnoid bypass, and foramen magnum decompression were all effective at reducing this pressure gradient.

Conclusions: Blockage of the spinal subarachnoid CSF pathway produces a relative increase in the pressure inside the spinal cord distal to the blockage point. Repetitive formation of this pressure gradient then induces CSF leakage into the spinal parenchyma, leading to the formation of syringomyelia. Using this model, alternative surgical procedures could be suggested that might be effective in treating this disease.
\end{abstract}

W ith the increasing availability of magnetic resonance imaging (MRI) of the spine, syringomyelia associated with adhesive arachnoiditis is now diagnosed more often. However, the mechanism of syrinx formation in this disease is still poorly understood, and the results of surgical treatment not satisfactory. ${ }^{2}$

Although several investigators have proposed hypotheses on the pathophysiology of syringomyelia associated with Chiari type I malformation, they did not deal with another type of syringomyelia-syringomyelia associated with adhesive arachnoiditis (SAA). Nevertheless, these two types of syringomyelia have many factors in common: both are associated with some derangement of CSF dynamics in the spine; the MRI findings and the operative findings of the syringes are similar in the two types; and they respond, at least in a short term, to syrinx shunting procedures. Therefore, it is reasonable to assume that there are some common pathophysiological processes underlying these two types of syringomyelia.

As can be seen in phase contrast cine-mode MRI studies of the spine, the CSF makes to and fro movements along the spinal canal, synchronising with the cardiac cycle..$^{3-5}$ These movements are blocked at the level where adhesive arachnoiditis is present. The syrinx associated with adhesive arachnoiditis usually develops in the spinal cord just distal to this blockage of CSF flow. ${ }^{6}$ In our previous paper, ${ }^{7}$ we constructed a theoretical model of CSF dynamics in the spine and proposed a hypothesis on the pathophysiology of syringomyelia associated with Chiari type I malformation. In the current study, we applied this model to analyse CSF dynamics and its derangements in SAA. In addition, we simulated various surgical procedures including some that are currently not standard for the treatment of SAA. Based on these analyses, we propose a hypothesis on the pathophysiology of SAA.

\section{METHODS \\ Modelling of CSF dynamics}

In this study, we used the same mathematical model of the spinal CSF dynamics as we used in our previous study of syringomyelia with Chiari I malformation. The detailed methods are described in our previous paper. ${ }^{7}$ Briefly, we constructed an electrical circuit model that simulated the dynamics of the CSF in the spine, based on a standard method of fluid dynamics. ${ }^{89}$ In this model, the electrical current represented the CSF flow; the electrical resistance represented the resistance to the CSF flow; and the electrical capacitance represented the compliance (or temporary fluid storage capacity) of the soft tissue such as the dura or the spinal cord. In fig 1, the electrical diagram is superimposed on the corresponding anatomical structures. As can be seen in the figure, the model consists of two arrays of serially connected resistors, which are then mutually connected with multiple capacitors. The resistor arrays represent two CSF pathways in the spine: the subarachnoid space around the spinal cord, and a CSF pathway inside the cord, which may be viewed as the central canal. Thus the voltage accumulated in the capacitor bridging the two resistor arrays corresponds to pressure gradient between inside and outside the spinal cord. For convenience, we will call these capacitors $\left(C_{1}\right.$ to $C_{9}$ in fig 1) "inside capacitors", and those capacitors representing the compliance of the dura and the root sleeves $\left(D_{1}\right.$ to $D_{9}$ in fig 1) "outside capacitors".

\section{Modelling of the pulsatile wave}

We were interested in the response of this system to a sudden increase in electrical voltage on the cranial ends, which simulated the pulsatile CSF waves coming from the cranial side. $^{3-5}$ According to the standard theory of electric circuit analysis, we formulated a set of ordinary differential

Abbreviation: SAA, syringomyelia associated with adhesive arachnoiditis 


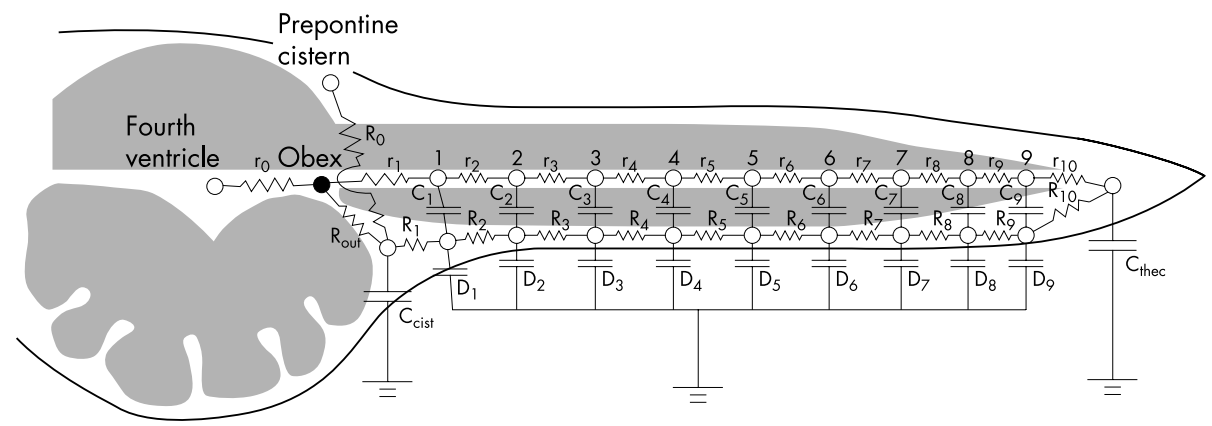

Figure 1 A schema showing our electric circuit model of the cerebrospinal fluid dynamics in the spine. The diagram is superimposed on corresponding anatomical structures. The upper row of resistors represents the central canal, whereas the lower row represents the spinal subarachnoid space. $C_{\text {cist }}$ represents the compliance of the cisterna magna, and $C_{\text {thec }}$ that of the lumbar thecal sac.

equations that described this electric circuit. We then solved these equations on a personal computer using a program for numerical analysis provided in a software package, Mathematica version 4.0 (Wolfram Research, Champaign, Illinois, USA). We used the same values of the resistors and capacitors as those in our previous study. The details are described in our previous paper. ${ }^{7}$

\section{Simulation of arachnoiditis and its treatment Arachnoiditis}

We then tried to simulate the situation in adhesive arachnoiditis by increasing the value of one of the resistors representing the spinal subarachnoid CSF pathway. In this report, we will show the result of increasing the value of the resistor $R_{5}$ (fig l) to 50 times the original value. We will call
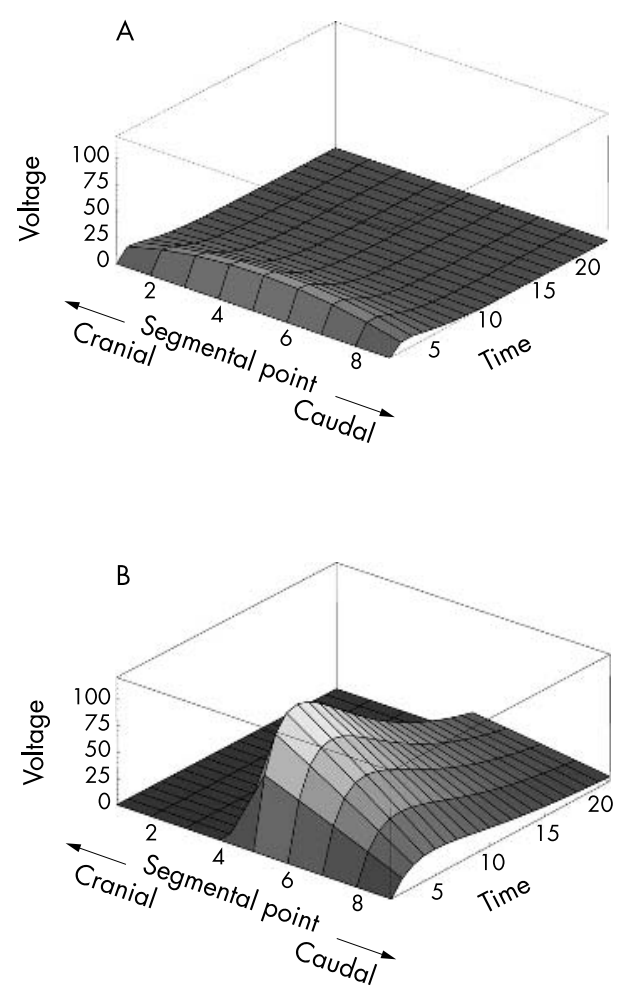

Figure 2 (A) The spatial distribution and time course of the voltage accumulated in the inside capacitors after a step input on the two cranial leads. (B) The same plot as $A$ except that the value of the resistor $R_{5}$ in fig 1 is increased to 50 times the original value, simulating adhesive arachnoiditis. this parameter setting the "original arachnoiditis setting," and refer to it later. The arbitrary selection of "50 times increase" is justified because we were only interested in the qualitative behaviour of the circuit, which was basically the same irrespective of the quantity of the increase.

\section{Syringo-subarachnoid shunt}

To simulate syringo-subarachnoid shunting, we added to the original arachnoiditis setting a resistor valued 0.2 between the central canal and the subarachnoid space, bypassing the capacitance $\mathrm{C}_{6}$ in fig $\mathrm{l}$. We then observed how this procedure affected the development of the pressure gradient distal to the blockage point.

\section{Subarachnoid bypass}

We then evaluated another mode of treatment that we called subarachnoid bypass, which was to bypass the blocked portion of the subarachnoid space with a catheter. To simulate this procedure, we added to the original arachnoiditis setting a resistor valued 0.2 between the subarachnoid space at point 4 and that at point 6 in fig 1 .

\section{Foramen magnum decompression}

In our previous paper, ${ }^{7}$ we hypothesised that the cisterna magna with its temporary fluid storage capacity functions as a shock absorber against the pulsatile CSF waves coming from the cranial side. Because foramen magnum decompression will increase the temporary fluid storage capacity of the cisterna magna, it will decrease the intensity of the pressure wave transmitted along the spinal cord. Therefore, it is a valid question whether foramen magnum decompression would be effective in treating SAA. We simulated foramen magnum

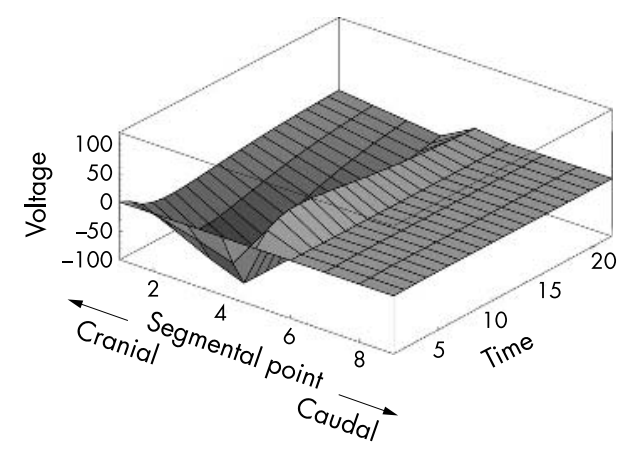

Figure 3 The same plot as in fig 2 except that an electrical shunt is added at the segment point 6 between the central canal and the subarachnoid space simulating a syringo-subarachnoid shunt. 


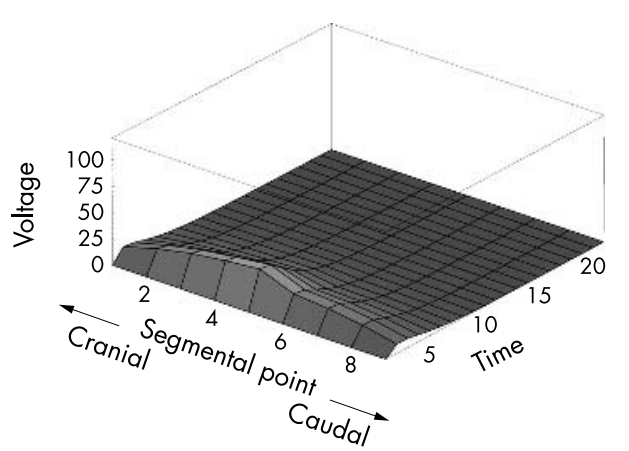

Figure 4 The same plot as in fig 2 except that an electrical shunt is added in the subarachnoid space between the segment point 4 and 6 bypassing the increased resistance at point 5 .

decompression by increasing the value of the capacitor $\mathrm{C}_{\text {cist }}$ (fig 1) to 10 times the original arachnoiditis setting. We calculated the response of this circuit to the same sudden increase of voltage on the cranial leads.

\section{RESULTS}

\section{Simulation of arachnoiditis}

Figure 2A shows the spatial distribution of the voltage accumulated in the inside capacitors $\left(C_{1}\right.$ to $\left.C_{9}\right)$, and its time course to a step increase of voltage on the two cranial leads. In our model, the voltages accumulated in the inside capacitors $\left(C_{1}\right.$ to $C_{9}$ in fig 1$)$ represent the pressure gradient between the inside and outside the spinal cord; a positive value indicates higher pressure inside. As shown in the figure, the intraspinal pressure was not increased in this setting.

Figure 2B shows the response of our circuit with the original arachnoiditis setting. Increasing the resistance of $R_{5}$ (fig l) produced increased pressure inside the spinal cord immediately caudal to the blockage point.

\section{Simulation of treatment}

Figure 3 shows the result of our simulation of syringosubarachnoid shunting. The insertion of the resistor simulating the syringo-subarachnoid shunt effectively reduced the increased pressure gradient produced by the arachnoiditis.

The result of the simulation of subarachnoid bypass procedure is shown in fig 4 . Shunting the rostral and caudal sides of the subarachnoid blockage effectively reduced the increased pressure inside the spinal cord shown in fig 2B.

Figure 5 shows the result of the simulation of foramen magnum decompression. This simulation was also effective in reducing the voltage accumulation shown in fig $2 \mathrm{~B}$.

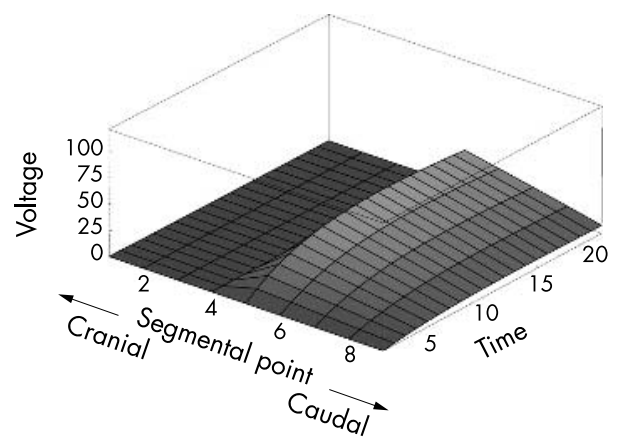

Figure 5 The same plot as in fig 2 except that the capacitance of the cisterna magna $\left(\mathrm{C}_{\text {cist }}\right.$ in fig 1$)$ is increased to 10 times its original value.

\section{DISCUSSION}

\section{Hypothesis}

The simulation of adhesive arachnoiditis with our model showed that the pressure inside the spinal cord is increased in the segment distal to the subarachnoid blockage. We can interpret this as follows (fig 6). Because of the CSF blockage in the subarachnoid space, the pulsatile CSF wave coming from above produces a pressure drop in the subarachnoid space distal to the blockage. In contrast, the pressure wave transmitted inside the spinal cord, presumably through the central canal or some other channel, is not hindered. Thus, in the segment distal to the blockage, the pressure inside the spinal cord becomes relatively larger than that outside. Repetitive formation of this pressure gradient at each CSF pulse leads to leakage of CSF from the central canal causing interstitial oedema and the formation of a syrinx. ${ }^{10}$

\section{Supporting evidence}

Our results are supported by clinical and experimental findings. Syringomyelia associated with adhesive arachnoiditis is most commonly located immediately distal to the adhesion, ${ }^{6}$ which corresponds to the location where we found the increased pressure gradient. Our results also closely paralleled the observation made by Klekamp et al in their animal model of SAA. ${ }^{11}$ They measured the pressure inside and outside the spinal cord in their kaolin soaked gel foam model of SAA, and found that the mean subarachnoid pressure distal to the adhesion was smaller than that on the cranial side. They also found that the pressure inside the spinal cord in the segment distal to the adhesion was relatively larger than that outside. This is exactly the result obtained in our analysis. The results of our simulation of syringo-subarachnoid shunting also correlate well with clinical findings. The effectiveness of syringo-subarachnoid shunting, which we demonstrated in our model, is well documented in the literature. ${ }^{212}{ }^{13} \mathrm{~A}$ recent case report by Takamura et al also supports our idea that increased intraspinal pressure distal to the adhesion leads to the formation of a syrinx. ${ }^{14}$ In that article, the investigators documented the development of syringomyelia associated with traumatic subarachnoid haemorrhage by serial MRI studies. They showed clearly how the initial enlargement of the central canal gradually developed into a full blown cervical syrinx. Subsequent surgery proved the presence of arachnoid adhesions at the craniovertebral junction. Simply interpreted, this report suggests that the central canal plays an important role in the formation of SAA.

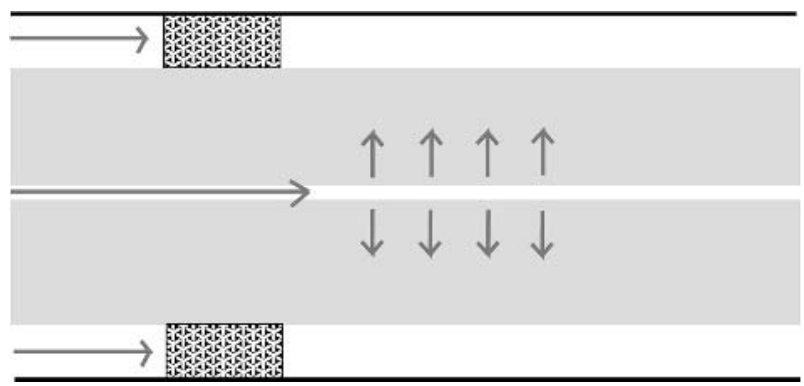

Figure 6 A schema of two fluid channels of the spine, one in the subarachnoid space, and the other inside the cord. By assuming the existence of these two channels, we can understand why the blockage of CSF in the subarachnoid space produces pressure gradient inside and outside the cord in the segment distal to the blockage. The arrows indicate the movement of the fluid. 


\section{Surgical treatment}

Although we have several surgical options for SAA, ${ }^{213}$ the results of those procedures are still not satisfactory. Syrinx shunting procedures showed high rates of malfunction and recurrence in long term follow up studies, ${ }^{12}{ }^{13}$ probably because of the progressive nature of the adhesive process. Although direct microsurgical dissection of the arachnoid scar may be the best surgical option,,$^{13} 16$ it is potentially dangerous ${ }^{17}$ in cases with severe arachnoid scarring.

Our study suggested the possible effectiveness of subarachnoid bypass procedures. It is quite understandable that bypassing the blockage of CSF flow, which restores the normal to and fro CSF movements to some extent, prevents the development of the pressure gradient by increasing the subarachnoid pressure distal to the blockage point. Although we have some anecdotal reports suggesting its effectiveness (personal communications), further clinical experience is needed to evaluate the role of this surgical procedure.

Our results also suggested the possible effectiveness of foramen magnum decompression on SAA. Currently, this procedure is certainly not a standard one. However, considering the progressive nature of this disease, its debilitating effect on the patient's quality of life, and the unsatisfactory results of the currently available surgical procedures, we may be justified if we considered its trial under strict ethical guidelines. If proven effective, foramen magnum decompression, being rather a safe procedure to undertake, could be a good alternative surgical procedure for SAA.

\section{Authors' affiliations}

H S Chang, H Nakagawa, Department of Neurological Surgery, Aichi Medical University, Aichi-gun, Japan

Competing interests: none declared

\section{REFERENCES}

1 Batzdorf U, Klekamp J, Johnson JP. A critical appraisal of syrinx cavity shunting procedures. J Neurosurg 1998;89:382-8.

2 Schaan M, Jaksche H. Comparison of different operative modalities in posttraumatic syringomyelia: preliminary report. Eur Spine J 2001;10:135-40.

3 Bering EA. Choroid plexus and arterial pulsation of cerebrospinal fluid. Demonstration of the choroids plexuses as a cerebrospinal fluid pump. Arch Neurol Psychiatry 1955;73:165-72.

4 Bhadelia RA, Bogdan AR, Kaplan RF, et al. Cerebrospinal fluid pulsation amplitude and its quantitative relationship to cerebral blood flow pulsations: a phase-contrast MR flow imaging study. Neuroradiology 1997;39:258-64.

5 Schroth G, Klose U. Cerebrospinal fluid flow. I. Physiology of cardiac-related pulsation. Neuroradiology 1992;35:1-9.

6 Inoue $Y$, Nemoto $Y$, Ohata K, et al. Syringomyelia associated with adhesive spinal arachnoiditis: MRI. Neuroradiology 2001;43:325-30.

7 Chang HS, Nakagawa $\mathrm{H}$. Hypothesis on the pathophysiology of syringomyelia based on simulation of cerebrospinal fluid dynamics. J Neurol Neurosurg Psychiatry 2003;74:344-7.

8 Segers $\mathbf{P}$, Stergiopulos $N$, Verdonck $P$, et al. Assessment of distributed arterial network models. Med Biol Eng Comput 1997;35:729-36.

9 Stergiopulos N, Young DF, Rogge TR. Computer simulation of arterial flow with applications to arterial and aortic stenoses. J Biomechanics 1992;25: 1477-88.

10 Levy El, Heiss JD, Kent MS, et al. Spinal cord swelling preceding syrinx development. Case report. J Neurosurg (Spine 1) 2000;92:93-7.

11 Klekamp J, Volkel K, Bartels CJ, et al. Disturbances of cerebrospinal fluid flow attributable to arachnoid scarring cause interstitial edema of the cat spinal cord. Neurosurgery 2001;48:174-86.

12 Iwasaki Y, Abe H. Surgical treatment for syringomyelia. No Shinkei Geka 1996;24:709-16.

13 Klekamp J, Batzdorf U, Samii M, et al. Treatment of syringomyelia associated with arachnoid scarring caused by arachnoiditis or trauma. J Neurosurg 1997;86:233-40.

14 Takamura Y, Kawasaki T, Takahashi A, et al. A craniocervical injury-induced syringomyelia caused by central canal dilation secondary to acquired tonsillar herniation. Case report. J Neurosurg 2001;95:122-7.

15 Lee TI, Arias JM, Andrus HL, et al. Progressive posttraumatic myelomalacic myelopathy: treatment with untethering and expansive duraplasty. J Neurosurg 1997;86:624-8.

16 Paramore CG. Dorsal arachnoid web with spinal cord compression: variant of an arachnoid cyst? Report of two cases. J Neurosurg 2000;93:287-90.

17 Isu T, Iwasaki Y, Akino M, et al. Surgical treatment of syringomyelia. Selection of surgical procedures. Neurol Med Chir 1989;29:728-34. 